\title{
The complementary value of intraoperative fluorescence imaging and Raman spectroscopy for cancer surgery: combining the incompatibles
}

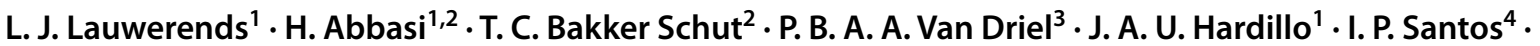 \\ E. M. Barroso ${ }^{5} \cdot$ S. Koljenović ${ }^{6} \cdot$ A. L. Vahrmeijer ${ }^{7} \cdot$ R. J. Baatenburg de Jong ${ }^{1} \cdot$ G. J. Puppels ${ }^{2} \cdot$ S. Keereweer ${ }^{1}$
}

Received: 4 November 2021 / Accepted: 23 January 2022 / Published online: 1 February 2022

(c) The Author(s) 2022

\begin{abstract}
A clear margin is an important prognostic factor for most solid tumours treated by surgery. Intraoperative fluorescence imaging using exogenous tumour-specific fluorescent agents has shown particular benefit in improving complete resection of tumour tissue. However, signal processing for fluorescence imaging is complex, and fluorescence signal intensity does not always perfectly correlate with tumour location. Raman spectroscopy has the capacity to accurately differentiate between malignant and healthy tissue based on their molecular composition. In Raman spectroscopy, specificity is uniquely high, but signal intensity is weak and Raman measurements are mainly performed in a point-wise manner on microscopic tissue volumes, making whole-field assessment temporally unfeasible. In this review, we describe the state-of-the-art of both optical techniques, paying special attention to the combined intraoperative application of fluorescence imaging and Raman spectroscopy in current clinical research. We demonstrate how these techniques are complementary and address the technical challenges that have traditionally led them to be considered mutually exclusive for clinical implementation. Finally, we present a novel strategy that exploits the optimal characteristics of both modalities to facilitate resection with clear surgical margins.
\end{abstract}

Keywords Raman spectroscopy $\cdot$ Fluorescence imaging $\cdot$ Image-guided surgery $\cdot$ Tumour differentiation $\cdot$ Resection margin assessment $\cdot$ Multimodal optical diagnostics

\section{Introduction}

Cancer is the leading cause of premature death in Europe and the USA and one of the most important public health issues worldwide [1,2]. Depending on the tumour type and stage, different treatment options are available. Surgery is the main treatment modality for most solid tumours, often integrated in a more extensive (neo-)adjuvant treatment strategy. Surgical resection is generally aimed at complete

This article is part of the Topical Collection on OncologyGeneral

S. Keereweer

s.keereweer@erasmusmc.nl

1 Department of Otorhinolaryngology, Head and Neck Surgery, Erasmus MC Cancer Institute, Rotterdam, Netherlands

2 Center for Optical Diagnostics and Therapy, Department of Dermatology, Erasmus MC Cancer Institute, Rotterdam, Netherlands

3 Department of Orthopedic Surgery, Isala Hospital, Zwolle, Netherlands
4 Molecular Physical-Chemistry R\&D Unit, Department of Chemistry, University of Coimbra, Coimbra, Portugal

5 AlfaRim Medical Holding, Delft, Netherlands

6 Department of Pathology, Antwerp University Hospital/Antwerp University, Antwerp, Belgium

7 Department of Surgery, Leiden University Medical Center, Leiden, Netherlands 
macroscopic and microscopic removal of the cancer. However, inadequate resection of the tumour (i.e. presence of cancer cells at the margin or $<5 \mathrm{~mm}$ from the resection surface) frequently occurs. The worldwide extent of this problem is illustrated in Table 1, showing the percentage of positive surgical margins for the most common cancer types. Although a clear surgical margin may not be pivotal in all cancer types (e.g. during debulking procedures for brain tumours [3]), it is the main prognostic factor for survival in most types of cancer. Moreover, inadequate surgical tumour resection most often warrants adjuvant treatment, which is associated with increased morbidity and costs. During the operation, an oncologic surgeon takes into account all relevant clinical information that is available, including cancer type, tumour differentiation, and preoperative imaging reports. However, the actual surgical cut is based on subjective tactile and visual assessment of the tissue to determine which tissue needs to be excised. Although the primary aim is to completely excise the tumour, precise margin delineation is imperative in many delicate areas where wider resections inevitably lead to increased morbidity and loss of functionality.

Over the past two decades, important advances have been made to improve assessment of surgical margins by the introduction of intraoperative imaging techniques. Besides frozen section analysis, several optical methods have been studied to perform intraoperative margin assessment in surgical oncology, including optical coherence tomography (OCT) [36], photoacoustic tomography, terahertz imaging, second harmonic generation, confocal microscopy, fluorescence (lifetime) imaging, autofluorescence imaging (AFI), narrow-band imaging, hyper-spectral imaging, diffuse reflectance spectroscopy, Fourier transform infrared spectroscopy, and Raman spectroscopy (RS) [37-42]. Among them, intraoperative fluorescence imaging (FLI) using exogenous tumour-specific fluorescent agents has shown to be particularly beneficial for surgical margin assessment in clinical trials [43-54], and it has been shown that RS can objectively discriminate between normal and malignant tissue [55-59]. Intraoperative FLI provides a wide-field realtime tumour-specific image to guide the surgical resection. However, detection and interpretation of the fluorescent signal are complex, and are influenced by optical tissue properties, targeting specificity of fluorescent agents and accuracy of the imaging system. Each of these factors has an effect on image resolution, sensitivity, and/or specificity of the technique [60]. RS on the other hand provides detailed identification of malignant tissues with high sensitivity and specificity [61]. It does not provide the surgeon with a wide-field image because it generally requires 'point' (i.e. of small tissue volumes) measurements of the tissue. This compromises the possibility of scanning the complete resection surface.

In this review, we describe the state-of-the-art of FLI and RS and include an overview of the advantages and limitations for their use in image-guided cancer surgery. Next, we demonstrate how these two techniques are complementary and how they can overcome each other's disadvantages and limitations. Finally, we highlight the technical challenges that need to be addressed to implement this novel approach into clinical practice.

\section{Image-guided cancer surgery using fluorescence imaging}

Intraoperative FLI of tumour tissue requires the systemic administration of a cancer-specific exogenous fluorescent agent that can identify the tumour using a spectrum of targeting strategies, including antibodies, small peptides, and activatable fluorophores [62-64]. The use of fluorophores that emit light in the first near-infrared (NIR-I) range $(650-900 \mathrm{~nm})$ results in deeper tissue penetration of the photons and lower autofluorescence from surrounding tissues [65-67]. Because the human eye is insensitive to light in this spectrum, dedicated intraoperative camera systems
Table 1 Percentage of positive surgical margins for the most common cancer types (estimated new cases worldwide, 2020). *Males and females combined

\begin{tabular}{lclc}
\hline Cancer type (solid tumours only) & $\begin{array}{l}\text { Estimated new } \\
\text { cases [4]* }\end{array}$ & Incidence [4]* & Positive margins \\
\hline Breast & $2,261,419$ & $12 \%$ & $20-70 \%[5-8]$ \\
Trachea, lung, and bronchus & $2,206,771$ & $11 \%$ & $5-17 \%[9-12]$ \\
Prostate & $1,414,259$ & $7 \%$ & $7-75 \%[13-16]$ \\
Colorectal & $1,931,590$ & $10 \%$ & $12-58 \%[17-19]$ \\
Urinary bladder & 573,278 & $3 \%$ & $0-25 \%[20-22]$ \\
Kidney and renal pelvis & 431,288 & $2 \%$ & $7-11 \%[23-26]$ \\
Uterine corpus & 417,367 & $2 \%$ & $4-17 \%[8,27]$ \\
Pancreas & 495,773 & $3 \%$ & $18-85 \%[28-31]$ \\
Thyroid & 586,202 & $3 \%$ & $10-11 \%[8,32,33]$ \\
Lip, oral cavity & 377,713 & $2 \%$ & $5-43 \%[34,35]$ \\
\hline
\end{tabular}


are required to detect the fluorescent signal [45, 68-71]. Figure 1 shows the clinical status of targeted fluorescent agents in the visible channel, NIR-I-700-nm channel, and NIR-I800-nm channel together with their excitation and emission wavelengths.

FLI exhibits several favourable characteristics for clinical translation. Preclinical studies have demonstrated low toxicity with high sensitivity and specificity for tumour detection using targeted fluorescent agents [62, 63, 72, 73]. Besides the minimal risks associated with the use of lasers in the imaging system, there are no safety issues for the clinicians because no ionising radiation is used, and the technique is capable of real-time detection without interfering with the surgical field. Importantly, the whole-field imaging facilitates analysis of the complete surgical margin and remaining wound bed.

The simultaneous development of clinically available fluorescent agents and intraoperative camera systems over the past decade has now positioned intraoperative FLI as a promising real-time detection modality for surgeons in oncology. Since the first clinical trial of FLI with tumourspecific NIR fluorescent agents [45], ground-breaking results have been reported in patients with various cancer types, consistently showing excellent safety records [43-54, 74]. Indocyanine green (ICG), a nonspecific and US Food and Drug Administration approved compound, has commonly been used for FLI in various settings [75, 76]. Methylene blue (MB) was approved as the second NIR fluorescent agent for fluorescence-based intraoperative imaging [77]. Fluorescein sodium, 5-ALA, and Cytalux/Pafolacianine are three other clinically approved fluorescent agents. To date, fluorescent agents [78, 79] have been developed for detection of cancer cells [80-83], sentinel lymph nodes [43, 71, 84, 85], atherosclerosis [86, 87], arthritis [88-90], ureters [91-93], bile ducts [94], and nerves [95, 96]. For visualisation of these fluorescent agents, specialised intraoperative imaging systems have been developed for open surgery [45, 46, 63, 68-70, 97, 98], endoscopy [99], laparoscopy [100], thoracoscopy [101], and robotic surgery [102, 103]. Figure 2 shows the intraoperative impact of using fluorescenceguided surgeries.

Despite a strong driving force coming from the auspicious results of these trials, there is still a fundamental controversy in the scientific field on how to cope with the complex process of optical imaging. Optical properties of the tissue can attenuate fluorescence signals; light scattering, for example, may cause blurred images. In addition, tumour-to-background ratios are influenced by nonspecific autofluorescence of the surrounding healthy tissues, although this disturbance is lower in the NIR region. Further, fluorescence intensity is further dependent on many factors, such as the concentration and fluorescence quantum yield of the fluorescent agent, the affinity of the fluorescent agent, the abundance of target receptors or epitopes, and the imaging system [60]. Innovative calculation methods have been developed that can partly correct for the perturbation caused by tissue optical properties, but a millimetre-scale sharp delineation of the tumour remains challenging in many cases [98, 104-107]. Considering all these factors, sufficient understanding of these processes by the surgeon is essential to adequately interpret the intraoperative fluorescence image at hand.

\section{Intraoperative use of Raman spectroscopy}

Raman spectroscopy is an optical technique that can be used for real-time characterisation of biological tissue in and ex vivo. A Raman spectrum of tissue is a representation of the molecular composition of that tissue. As cancer alters the molecular composition of tissue, RS can be used for discrimination between tumour and healthy tissue. Contrary to FLI, the technique does not require preparation or staining of the tissue to be analysed, which facilitates clinical application. With the use of optical fibres, many body locations can be assessed in vivo using hand-held RS probes [113]. RS has a high diagnostic accuracy for cancer detection, with reported overall diagnostic sensitivities and specificities between 73 and 100\% and 66 and 100\%, respectively [114]. Its intraoperative application has recently been explored in brain tumour biopsies [115] and brain tumour surgery [116, 117], as well as in debulking procedures of ovarian cancer [118]. RS can further be used for peripheral nerve visualisation and identification and sparing of vital structures during oncological surgery $[119,120]$.

The two regions of the Raman spectrum that are relevant to cancer diagnostics are the fingerprint and the high wavenumber (HWVN) regions (Fig. 3d, e). Most biomedical research on RS studies the fingerprint region $\left(\sim 400-2000 \mathrm{~cm}^{-1}\right)$. This region is rich in spectral features, including the biochemical signatures associated with lipids, proteins, nucleic acids (DNA), and blood [121]. However, processing times in this region are long; signal intensity is relatively low, and molecular discrimination is generally based on minute spectral differences, necessitating complex and robust machine learning algorithms [61, 122]. Furthermore, in vivo applications for RS require fibre optic probes. These fibres, made of fused silica, generate intense background emission requiring complex optical filtering [123]. Contrary to the fingerprint region, no Raman signal is generated in fused silica in the HWVN region, which ranges from $\sim 2400$ to $3800 \mathrm{~cm}^{-1}$. In addition to its amenability for use with optical fibres, the signal in the HWVN region is much more intense than in the fingerprint region. While it contains less detailed spectral information, the HWVN region can be utilised to distinguish malignant from healthy 

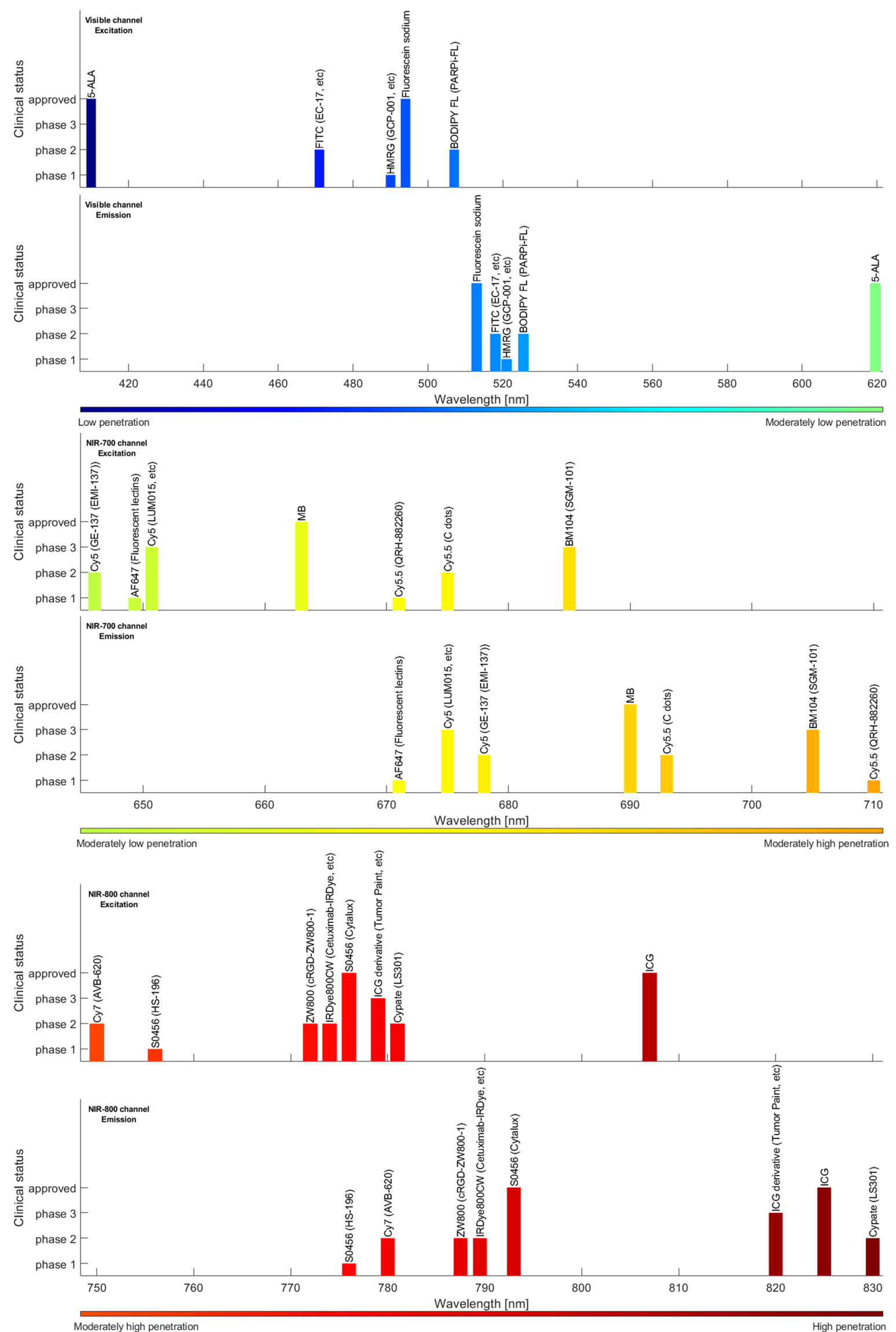
4Fig. 1 Excitation and emission wavelengths of approved and clinical fluorescent agents in the visible channel, NIR-700-nm channel, and NIR-800-nm channel (data collected from [108-112])

tissue with a more straightforward approach. An additional benefit of performing RS in the HWVN over the fingerprint region is the greater spectral distance from fluorescence emission, when excited with a red or NIR laser. As a result, the Raman signal in the HWVN region experiences less interference with that of fluorescence as compared to the fingerprint region.

In oral cancer, it has been demonstrated that HWVN Raman spectra can identify cancer ex vivo based on the water concentration in freshly excised specimens [55]. This water concentration differs consistently across the border between tumour and healthy surrounding tissue over a distance of about $5 \mathrm{~mm}$ [124]. The water concentration averages from $76 \%$ in the tumour to about $54 \%$ in the surrounding healthy tissue. The variation in water concentration inside tumour tissue appeared to be much lower $(8 \%)$ than that in the healthy tissue (24\%) [124]. Acquisition of HWVN Raman spectra occurred in a point-based manner, i.e. grid-wise measurements of microscopic tissue volumes with instant signal processing $(<0.1 \mathrm{~s})$.

Based on these findings, the development of an automated fibre optic needle probe for determination of the water concentration profile across the margins of a resection specimen has been initiated. The needle probe is placed on the resection surface and then driven into the tissue while recording spectra at regular distance intervals along its path. The goal is to measure the distance between resection surface and tumour border in this way, to enable rapid and objective margin assessment without the need for specimen grossing [125-127manuscript in preparation Aaboubout et al.].

The combination of other imaging modalities with selective sampling of suspicious areas by RS as a powerful labelfree analytical technique has also been explored [128, 129]. Such approaches include MRI, second harmonic generation, OCT, AFI, total internal reflection fluorescence, or quantitative phase microscopy [130-138]. In general, the basic concept behind these multimodal approaches is to combine the high sensitivity of an imaging modality (i.e. rapid identification of areas that need further inspection) with the high selectivity of RS tissue analysis.

\section{The potential complementary value of combined fluorescence imaging and Raman spectroscopy}

In FLI, the signal originates from the interaction of light with the administrated fluorescent agent binding in the tumour. In RS, however, the signal originates from the interaction of light with the tissue itself, regardless of the specificity of fluorescent agent binding. Owing to the distinctly different physical signal origins of FLI and RS, the provided information is complementary.

FLI allows for real-time whole-field imaging of the surgical field, with high signal intensity. However, fluorescence signal intensity is limited by intrinsic optical tissue properties as well as the concentration and fluorescent quantum yield of the fluorescent agent [60]. On the other hand, RS can detect tumour with uniquely high specificity, but the signal intensity is very weak and can be hindered by photon shot noise originating from light interaction with components of the specimen present in the cross-section of the illumination and detection paths [139]. Additionally, its limitation to point measurements does not allow for real-time, whole-field tissue analysis. Therefore, a combination of FLI that has high sensitivity at the expense of a somewhat lower specificity, with RS that provides high specificity would be very advantageous. FLI would be employed in vivo for real-time identification of fluorescence, followed by objective verification of malignancy with RS to yield superior diagnostic accuracy over either modality used in isolation.

Other than helping to distinguish malignant from healthy tissue (i.e. during excision with tumour-free margins and debulking procedures), FLI has additional advantages for surgical oncology. Due to its ability to scan large surfaces, FLI is a useful tool for identifying additional occult lesions as well as relevant vital structures that require preservation during cancer surgery [140]. FLI has been employed to identify separately located clinically occult tumours in a variety of contexts, including peritoneal metastases [141], sentinel lymph nodes [142], pulmonary [143], and abdominal [144] lesions. Such findings have been described to result in improved survival rates, changes in personalised treatment, and reduced morbidity associated with damage to healthy surrounding structures.

Besides securing adequate margins and achieving conservative resection during cancer surgery, RS can complement FLI in several other ways. When fluorescent areas are detected in the wound bed or elsewhere in the scanned surface (e.g. abdomen), RS could be the ideal tool to instantly assess this suspect region, thereby confirming or ruling out the presence of occult lesions. For unanticipated intraoperative findings such as the detection of clinically occult peritoneal metastases during colorectal cancer surgery [141], subsequent in vivo RS could be used to confirm malignancy without the need for time-consuming fresh frozen sectioning. Even in case of a clinically suspicious non-fluorescent lesion, RS can provide timely analysis. Similarly, RS has potential for use as an additional confirmation of fluorescent findings during debulking procedures and in the identification of vital structures. 


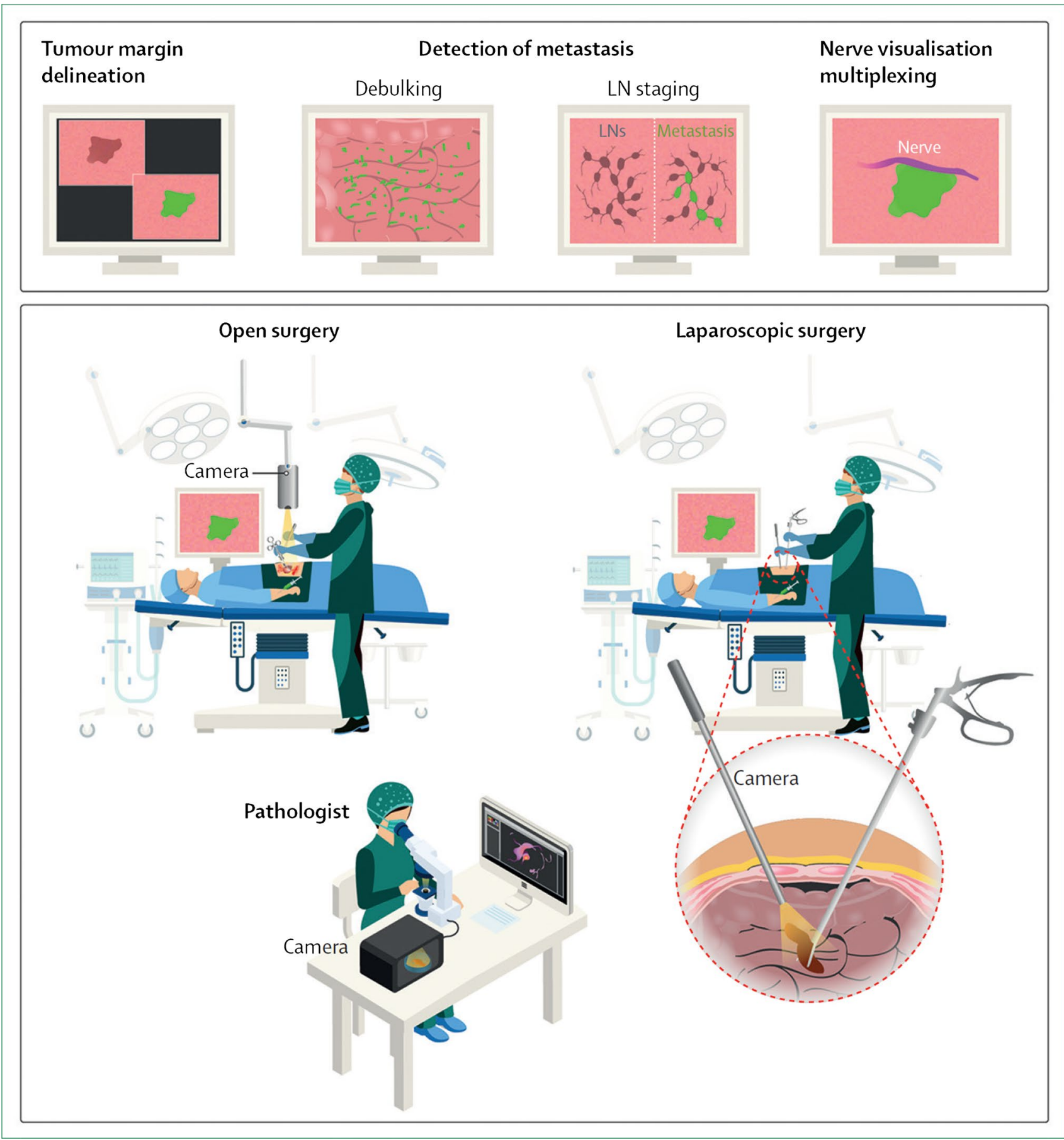

Fig. 2 (Top) Schematic illustration of the intraoperative impact of using fluorescence-guided surgeries. (Bottom) Implementation of fluorescence guidance. Following the administration of the fluorescent agent, the tissues of interest can be visualised with a dedicated NIR camera in real-time during open or laparoscopic surgery. In addition

However, laser-induced fluorescence itself can be a limiting factor for Raman measurements. Because fluorescent signals are generally orders of magnitude stronger, obscuring the Raman signal, these techniques were traditionally to guiding the surgeon during the resection, rapid feedback on the presence of fluorescence can be provided by imaging the resected tissue on a back table with a NIR camera and microscope in the operating room (taken from [79] with permission)

considered mutually exclusive. Figure 3 shows this interference in preliminary tests where RS was performed utilising the standard excitation wavelength of $671 \mathrm{~nm}$ on freshly excised tissue of a patient after injection of a fluorescent 

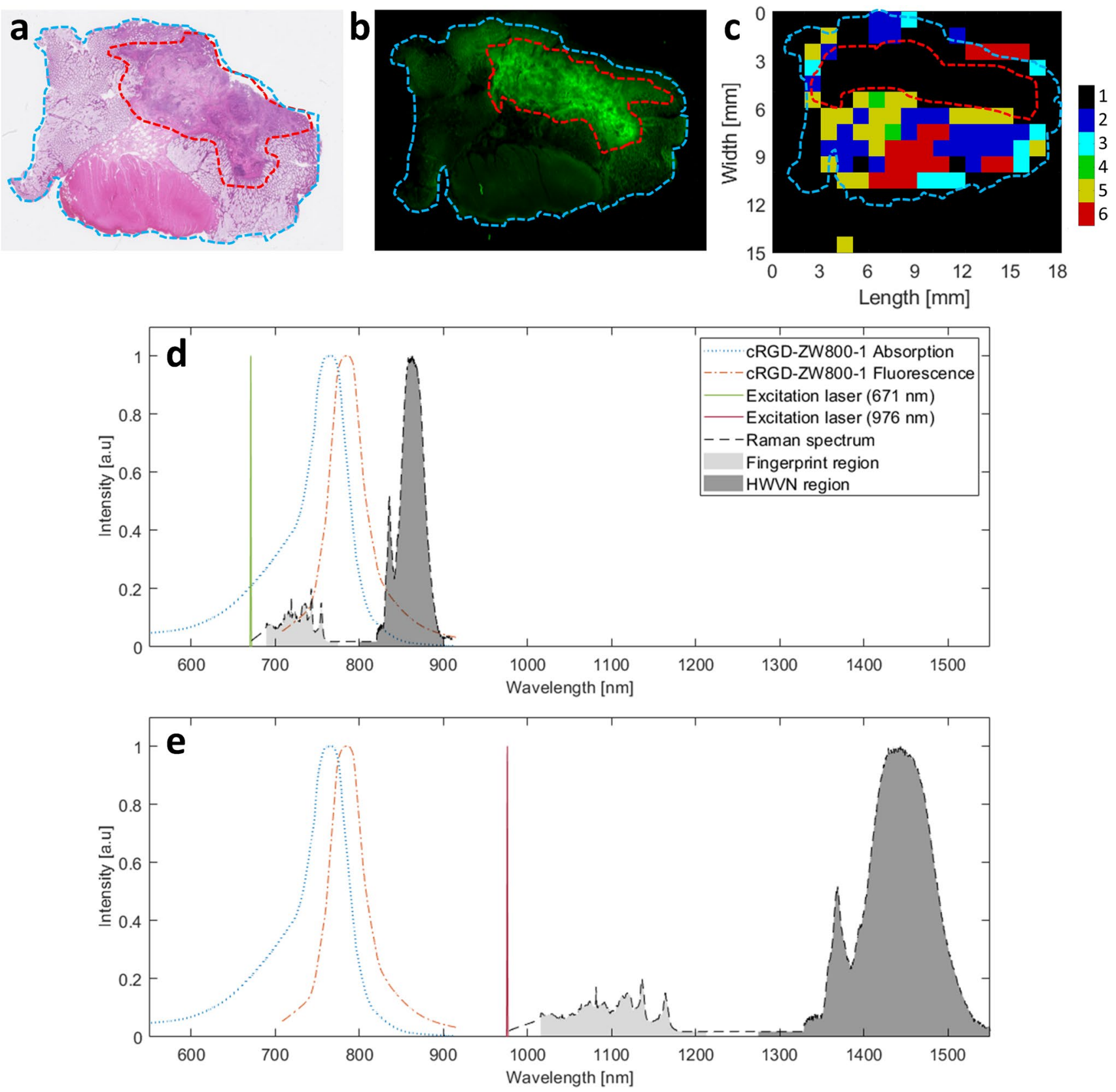

Fig. 3 FLI and RS image of tissue section of colorectal cancer containing cRGD-ZW800-1 and surrounding healthy tissue. Ethical approval for collection of this tissue falls under the METC LUMC, as part of studies registered in the European Trials Database under numbers 2016-000,397-38 and 2017-002,772-60 [73]. Tumour is delineated by the red dotted line; tissue is delineated by blue dotted line ( $a, b$, and c). a Haematoxylin and eosin-stained slide. b Fluorescent image showing colocalisation of signal with tumour. $\mathbf{c}$ Cluster

agent in the NIR-800-nm channel. The interface can be eliminated by shifting the excitation wavelength to $976 \mathrm{~nm}$.

Combining FLI with RS indeed requires careful matching of excitation and emission wavelengths of RS and the exogenous fluorescent agents; several groups have studied analysis of the Raman image illustrating that the tumour area cannot be characterised by RS because the fluorescence signal is too strong. d Fluorescence spectrum of cRGD-ZW800-1 showing overlap with the acquired Raman signal at an excitation wavelength of $671 \mathrm{~nm}$. HWVN region provides stronger Raman signal intensity than other Raman regions and overlaps with the tail of fluorescence emission. e Space between fluorescence spectrum of cRGD-ZW800-1 and the acquired Raman signal at an excitation wavelength of $976 \mathrm{~nm}$

various approaches to deal with this issue. The excitation of fluorescent labels can be avoided by shifting the wavelength of the Raman laser to a longer wavelength [145].

In a study using phantoms and breast specimens stained with patent blue dye (BD), it was demonstrated that 
fluorescence induction from surgical pigments could be circumvented by combining a laser with a longer wavelength $(785 \mathrm{~nm}$ vs. $685 \mathrm{~nm})$ with an indium gallium arsenide (InGaAs) camera capable of measuring the water/total area ratio of the HWVN spectrum [146]. Noting that haemoglobin and $\mathrm{BD}$ have no adverse effect on water content analysis, the Raman system was shown to accurately distinguish tumour from healthy tissue, as well as differentiate between normal and metastatic axillary lymph nodes in the presence of BD [147].

Another study combined fingerprint RS with wide-field AFI spectral imaging for intraoperative margin assessment in breast-conserving surgery [130]. AFI was employed to effectively 'screen out' the adipose tissue, enabling RS to more efficiently target the non-adipose tissue regions that are at a higher risk of malignancy, with superior diagnostic value as a result.

When it comes to the combination of FLI and RS, 5-aminolevulinic acid (5-ALA) is the most frequently studied fluorescent agent. In 2006, in vivo fingerprint RS was studied on bladder cancer biopsies obtained under the guidance of 5-ALA-induced protoporphyrin IX (PPIX) FLI [148]. Although a NIR laser emitting at $830 \mathrm{~nm}$ was used to reduce the interference of PPIX fluorescence emission in the visible area, additional fluorescence background was observed in the sample obtained from fluorescent areas, obscuring the RS signal and requiring algorithmic removal. Therefore, a new classification algorithm was trained based on biopsies with 5-ALA, which improved accuracy (from sensitivity and specificity of $43 \%$ and $71 \%$, to $75 \%$ and $89 \%$, respectively) [149]. Furthermore, it was demonstrated how the classification accuracy could be further improved by combining fluorescence and RS information on biopsies with 5-ALA (sensitivity and specificity of $100 \%$ and $81 \%$, respectively).

More recently, ex vivo RS was performed to differentiate glioma from normal brain in the presence of 5-ALA [150]. It was shown that RS could identify glioma with an accuracy of $0.85-1.00$ (along with a 95\% confidence interval) using a 785-nm laser and collecting Raman spectra in the fingerprint region. In optical tissue phantoms, tumour margin could be delineated using this approach [151]. Additionally, fingerprint RS was studied on tissues containing verteporfin and temoporfin, two fluorescent agents (photosensitisers) with activation at $\sim 690$ and $\sim 650 \mathrm{~nm}$, respectively [152]. With fluorescence emission similar to PPIX (600-800 nm), both agents were shown to be compatible with a 785 -nm excitation Raman system. However, no clinical in vivo intraoperative studies have been reported yet.

To summarise, several studies showed successful Raman measurements in the presence of fluorescent agents with emission in the visible and NIR-700-nm channel regions. However, to the best of our knowledge, there is no report on performing Raman measurements with the agents in the
NIR-800-nm channel (e.g. ICG). ICG is the most widely used fluorescent agent in clinical trials of fluorescenceguided surgery (FGS) [43, 153]. It has peak fluorescence emission in the NIR-I region and a long emission tail that extends into the NIR-II (1000-1700 nm) region [154], which is very challenging to combine with RS because of reduced Raman scattering at higher wavelengths, as well as the limited quantum efficiency of silicon-based CCD detectors, which declines to below $30 \%$ beyond $1000 \mathrm{~nm}$.

A novel HWVN RS system was recently developed to characterise tumour in highly pigmented skin lesions (i.e. melanomas) [155-157]. These melanocytic lesions have absorption characteristics at long wavelengths, similar to NIR fluorophores. Tissue characterisation was still feasible after increasing the laser excitation wavelen161. gth to/ beyond the NIR region (>900 nm), demonstrating that the combined and simultaneous use of FGS with NIR fluorophores and RS is technically feasible. Using this HWVN RS system, resection margins can be improved by utilising the optimal characteristics of FLI in the NIR region with tumour-specific fluorescent agents, combined with the specificity of RS based on differences in water concentration in this region. A laser excitation wavelength of $976 \mathrm{~nm}$ in combination with a novel low-noise InGaAs detector showed perfect elimination of laser-induced fluorescence of pigmented tissues. Collection of Raman signal in the shortwave infrared (SWIR) region with a high quantum efficiency (>90\%) up to $1570 \mathrm{~nm}$ showed minimal fluorescence contribution in the obtained spectra excited with 976-nm laser. RS with a low-noise InGaAs multichannel detector in the SWIR region can open up possibilities of combining RS and FLI in a clinical, intraoperative setting, even in the presence of fluorescent agents in the NIR-800-nm channel.

Although RS is generally a label-free method, systemic injection of molecular imaging agents for Raman signal enhancement has recently been studied preclinically for margin assessment purposes. By selectively accumulating in the tumour tissue, multi-purpose nanoparticles could be used for FLI while simultaneously enhancing the Raman signal, as well as providing the option of photothermal tumour ablation [158-160]. This promising approach still awaits clinical translation.

\section{Conclusion and future work}

While combining FLI with RS to achieve both whole-field imaging of the surgical field and objective margin verification would be very advantageous, these techniques have traditionally been considered mutually exclusive. Careful matching of RS excitation wavelengths and fluorophore emission wavelengths is required, presenting various technical hurdles. This issue could be solved by quantification 
of differences in water concentration in the HWVN region. After FLI with tumour-specific fluorescent agents, HWVN RS may be used to assess margin adequacy. In addition, evidence for the utility of RS in the identification of occult lesions and vital structures underlines its potential as an adjunct to FGS in all its basic applications. It is worth mentioning that intraoperative imaging techniques can also be beneficial for other applications, not limited to cancer surgery. These include delineation of targeted borders for precise surgical resections, evaluation of vascular perfusion, and identification of vital structures, such as nerves or ureters [140]. The synergistic effect of FLI and RS could also be exploited for these applications.

Author contribution The idea for the manuscript was proposed by $\mathrm{S}$. Keereweer. The scope of it was discussed by all authors. S. Keereweer, L.J. Lauwerends, and H. Abbasi performed the literature search and wrote the first draft. The manuscript was critically read, and feedback was provided by all authors. All authors approved the final manuscript.

Funding This work was supported by the Dutch Cancer Society KWF [Research Grant: 12175] and the Daniel den Hoed Fonds.

\section{Declarations}

Ethics approval Not applicable.

Conflict of interest The authors declare no competing interests.

Open Access This article is licensed under a Creative Commons Attribution 4.0 International License, which permits use, sharing, adaptation, distribution and reproduction in any medium or format, as long as you give appropriate credit to the original author(s) and the source, provide a link to the Creative Commons licence, and indicate if changes were made. The images or other third party material in this article are included in the article's Creative Commons licence, unless indicated otherwise in a credit line to the material. If material is not included in the article's Creative Commons licence and your intended use is not permitted by statutory regulation or exceeds the permitted use, you will need to obtain permission directly from the copyright holder. To view a copy of this licence, visit http://creativecommons.org/licenses/by/4.0/.

\section{References}

1. Siegel RL, Miller KD, Jemal A. Cancer statistics, 2018. CA Cancer J Clin. 2018;68(1):7-30.

2. Bray F, et al. Global cancer statistics 2018: GLOBOCAN estimates of incidence and mortality worldwide for 36 cancers in 185 countries. CA Cancer J Clin. 2018;68(6):394-424.

3. Eyupoglu IY, Buchfelder M, Savaskan NE. Surgical resection of malignant gliomas-role in optimizing patient outcome. Nat Rev Neurol. 2013;9(3):141-51.

4. Sung H, et al. Global Cancer Statistics 2020: GLOBOCAN estimates of incidence and mortality worldwide for 36 cancers in 185 countries. CA Cancer J Clin. 2021;71(3):209-49.

5. Jacobs L. Positive margins: the challenge continues for breast surgeons. Ann Surg Oncol. 2008;15(5):1271-2.
6. Miller AR, et al. Positive margins following surgical resection of breast carcinoma: analysis of pathologic correlates. J Surg Oncol. 2004;86(3):134-40.

7. Pleijhuis RG, et al. Obtaining adequate surgical margins in breast-conserving therapy for patients with early-stage breast cancer: current modalities and future directions. Ann Surg Oncol. 2009;16(10):2717-30.

8. Orosco RK, et al. Positive surgical margins in the 10 most common solid cancers. Sci Rep. 2018;8(1):5686.

9. Magdeleinat $P$, et al. Surgical treatment of lung cancer invading the chest wall: results and prognostic factors. Ann Thorac Surg. 2001;71(4):1094-9.

10. Osarogiagbon RU, et al. Prevalence, prognostic implications, and survival modulators of incompletely resected non-small cell lung cancer in the U. S. National Cancer Data Base. J Thorac Oncol. 2016;11(1):e5-16.

11. Tandberg DJ, et al. Patterns of failure after surgery for nonsmall-cell lung cancer invading the chest wall. Clin Lung Cancer. 2017;18(4):e259-65.

12. Predina JD, et al. Clinical implications of positive margins following non-small cell lung cancer surgery. J Surg Oncol. 2016;113(3):264-9.

13. Bellangino $\mathbf{M}$, et al. Systematic review of studies reporting positive surgical margins after bladder neck sparing radical prostatectomy. Curr Urol Rep. 2017;18(12):99.

14. Shipley WU, et al. Radiation with or without antiandrogen therapy in recurrent prostate cancer. N Engl J Med. 2017;376(5):417-28.

15. Srougi V, et al. Surgical method influences specimen margins and biochemical recurrence during radical prostatectomy for high-risk prostate cancer: a systematic review and meta-analysis. World J Urol. 2017;35(10):1481-8.

16. Yossepowitch $\mathrm{O}$, et al. Positive surgical margins after radical prostatectomy: a systematic review and contemporary update. Eur Urol. 2014;65(2):303-13.

17. Nuyttens JJ, et al. High-dose-rate intraoperative radiotherapy for close or positive margins in patients with locally advanced or recurrent rectal cancer. Int J Radiat Oncol Biol Phys. 2004;58(1):106-12.

18. Alberda WJ, et al. The importance of a minimal tumor-free resection margin in locally recurrent rectal cancer. Dis Colon Rectum. 2015;58(7):677-85.

19. Alberda WJ, et al. Intraoperative radiation therapy reduces local recurrence rates in patients with microscopically involved circumferential resection margins after resection of locally advanced rectal cancer. Int J Radiat Oncol Biol Phys. 2014;88(5):1032-40.

20. DiLizia EM, Sadeghi F. Surgical and pathological outcomes of robotic-assisted radical cystectomy for bladder cancer in the community setting. J Robot Surg. 2017.

21. Yuh B, et al. Systematic review and cumulative analysis of oncologic and functional outcomes after robot-assisted radical cystectomy. Eur Urol. 2015;67(3):402-22.

22. Novara $G$, et al. Soft tissue surgical margin status is a powerful predictor of outcomes after radical cystectomy: a multicenter study of more than 4,400 patients. J Urol. 2010;183(6):2165-70.

23. Seisen T, et al. A systematic review and meta-analysis of clinicopathologic factors linked to intravesical recurrence after radical nephroureterectomy to treat upper tract urothelial carcinoma. Eur Urol. 2015;67(6):1122-33.

24. Kryvenko ON. Positive surgical margins increase risk of recurrence after partial nephrectomy for high risk renal tumors. Shah PH, Moreira DM, Okhunov Z, Patel VR, Chopra S, Razmaria AA, Alom M, George AK, Yaskiv O, Schwartz MJ, Desai M, Vira MA, Richstone L, Landman J, Shalhav AL, Gill I, Kavoussi 
LR. J Urol. 2016 Aug;196(2):327-34. Urol Oncol. 2017; 35 (6): 449-450.

25. Ani I, et al. Prevalence and impact on survival of positive surgical margins in partial nephrectomy for renal cell carcinoma: a population-based study. BJU Int. 2013;111(8):E300-5.

26. Alemozaffar M, Filson CP, Master VA. The importance of surgical margins in renal cell and urothelial carcinomas. J Surg Oncol. 2016;113(3):316-22.

27. Seagle BL, et al. Survival after pelvic exenteration for uterine malignancy: a National Cancer Data Base study. Gynecol Oncol. 2016;143(3):472-8.

28. Handgraaf $\mathrm{HJ}$, et al. Current and future intraoperative imaging strategies to increase radical resection rates in pancreatic cancer surgery. Biomed Res Int. 2014;2014:890230.

29. Menon KV, et al. Impact of margin status on survival following pancreatoduodenectomy for cancer: the Leeds Pathology Protocol (LEEPP). HPB (Oxford). 2009;11(1):18-24.

30. Verbeke CS, Gladhaug IP. Resection margin involvement and tumour origin in pancreatic head cancer. Br J Surg. 2012;99(8):1036-49.

31. Esposito I, et al. Most pancreatic cancer resections are R1 resections. Ann Surg Oncol. 2008;15(6):1651-60.

32. Kluijfhout WP, et al. Microscopic positive tumor margin does not increase the risk of recurrence in patients with T1-T2 well-differentiated thyroid cancer. Ann Surg Oncol. 2016;23(5):1446-51.

33. Wang LY, et al. Microscopic positive margins in differentiated thyroid cancer is not an independent predictor of local failure. Thyroid. 2015;25(9):993-8.

34. Smits RW, et al. Resection margins in oral cancer surgery: room for improvement. Head Neck. 2016;38(Suppl 1):E2197-203.

35. McMahon $\mathrm{J}$, et al. Influence of condition of surgical margins on local recurrence and disease-specific survival in oral and oropharyngeal cancer. Br J Oral Maxillofac Surg. 2003;41(4):224-31.

36. van Manen L, et al. The clinical usefulness of optical coherence tomography during cancer interventions. J Cancer Res Clin Oncol. 2018;144(10):1967-90.

37. Brouwer de Koning SG, Schaeffers AWMA, Schats W, van den Brekel MWM, Ruers TJM, Karakullukcu MB. Assessment of the deep resection margin during oral cancer surgery: a systematic review. Eur J Surg Oncol. 2021;47(9):2220-32. https://doi.org/ 10.1016/j.ejso.2021.04.016.

38. Balasundaram $\mathrm{G}$, et al. Biophotonic technologies for assessment of breast tumor surgical margins-a review. J Biophotonics. 2021;14(1):e202000280.

39. Lauwerends LJ, et al. Current intraoperative imaging techniques to improve surgical resection of laryngeal cancer: a systematic review. Cancers. 2021;13(8):1895.

40. Shipp DW, et al. Intra-operative spectroscopic assessment of surgical margins during breast conserving surgery. Breast Cancer Res. 2018;20(1):69.

41. Heidkamp J, et al. Novel imaging techniques for intraoperative margin assessment in surgical oncology: a systematic review. Int J Cancer. 2021;149(3):635-45.

42. Sachańbiński T, Radecka B. Review of methods of intraoperative margin assessment in breast conserving surgery. Nowotwory. $\mathrm{J}$ Oncol. 2021; 0(0).

43. Schaafsma BE, et al. The clinical use of indocyanine green as a near-infrared fluorescent contrast agent for image-guided oncologic surgery. J Surg Oncol. 2011;104(3):323-32.

44. Hutteman M, et al. Near-infrared fluorescence imaging in patients undergoing pancreaticoduodenectomy. Eur Surg Res. 2011;47(2):90-7.
45. van Dam GM, et al. Intraoperative tumor-specific fluorescence imaging in ovarian cancer by folate receptor-alpha targeting: first in-human results. Nat Med. 2011;17(10):1315-9.

46. Vahrmeijer AL, et al. Image-guided cancer surgery using nearinfrared fluorescence. Nat Rev Clin Oncol. 2013;10(9):507-18.

47. van der Vorst JR, et al. Near-infrared fluorescence-guided resection of colorectal liver metastases. Cancer. 2013;119(18):3411-8.

48. Handgraaf HJM, et al. Real-time near-infrared fluorescence imaging using cRGD-ZW800-1 for intraoperative visualization of multiple cancer types. Oncotarget. 2017;8(13):21054-66.

49. Handgraaf HJM, et al. Long-term follow-up after near-infrared fluorescence-guided resection of colorectal liver metastases: a retrospective multicenter analysis. Eur J Surg Oncol. 2017;43(8):1463-71.

50. Boogerd LSF, et al. Safety and effectiveness of SGM-101, a fluorescent antibody targeting carcinoembryonic antigen, for intraoperative detection of colorectal cancer: a dose-escalation pilot study. Lancet Gastroenterol Hepatol. 2018;3(3):181-91.

51. Hekman MC, et al. Tumor-targeted dual-modality imaging to improve intraoperative visualization of clear cell renal cell carcinoma: a first in man study. Theranostics. 2018;8(8):2161-70.

52. van Keulen $\mathbf{S}$, et al. The clinical application of fluorescence-guided surgery in head and neck cancer. J Nucl Med. 2019;60(6):758-63.

53. Voskuil FJ, et al. Exploiting metabolic acidosis in solid cancers using a tumor-agnostic $\mathrm{pH}$-activatable nanoprobe for fluorescence-guided surgery. Nat Commun. 2020;11(1):3257.

54. Schaap DP, et al. Carcinoembryonic antigen-specific, fluorescent image-guided cytoreductive surgery with hyperthermic intraperitoneal chemotherapy for metastatic colorectal cancer. Br J Surg. 2020;107(4):334-7.

55. Barroso EM, et al. Discrimination between oral cancer and healthy tissue based on water content determined by Raman spectroscopy. Anal Chem. 2015;87(4):2419-26.

56. Liao Z, et al. Feasibility of integrated high-wavenumber Raman imaging and fingerprint Raman spectroscopy for fast margin assessment in breast cancer surgery. J Raman Spectrosc. 2020;51(10):1986-95.

57. Pence IJ, et al. Discrimination of liver malignancies with 1064 $\mathrm{nm}$ dispersive Raman spectroscopy. Biomed Opt Express. 2015;6(8):2724-37.

58. Thomas G, et al. Evaluating feasibility of an automated 3-dimensional scanner using Raman spectroscopy for intraoperative breast margin assessment. Sci Rep. 2017;7(1):13548.

59. Haifler, M. et al. Discrimination of malignant and normal kidney tissue with short wave infrared dispersive Raman spectroscopy. J Biophotonics 2018; 11(6): p. e201700188.

60. Keereweer S, et al. Optical image-guided cancer surgery: challenges and limitations. Clin Cancer Res. 2013;19(14):3745-54.

61. Koljenovic $\mathrm{S}$, et al. Tissue characterization using high wave number Raman spectroscopy. J Biomed Opt. 2005;10(3):031116.

62. Keereweer S, et al. Optical image-guided surgery-where do we stand? Mol Imaging Biol. 2011;13(2):199-207.

63. Zhang RR, et al. Beyond the margins: real-time detection of cancer using targeted fluorophores. Nat Rev Clin Oncol. 2017;14(6):347-64.

64. Mieog JSD, Achterberg FB, Zlitni A, Hutteman M, Burggraaf J, Swijnenburg RJ, Gioux S, Vahrmeijer AL. Fundamentals and developments in fluorescence-guided cancer surgery. Nat Rev Clin Oncol. 2022;19(1):9-22. https://doi.org/10.1038/ s41571-021-00548-3.

65. Frangioni JV. In vivo near-infrared fluorescence imaging. Curr Opin Chem Biol. 2003;7(5):626-34.

66. Frangioni JV. New technologies for human cancer imaging. J Clin Oncol. 2008;26(24):4012-21. 
67. Weissleder R, Ntziachristos V. Shedding light onto live molecular targets. Nat Med. 2003;9(1):123-8.

68. Mieog JS, et al. Novel intraoperative near-infrared fluorescence camera system for optical image-guided cancer surgery. Mol Imaging. 2010;9(4):223-31.

69. Troyan SL, et al. The FLARE intraoperative near-infrared fluorescence imaging system: a first-in-human clinical trial in breast cancer sentinel lymph node mapping. Ann Surg Oncol. 2009;16(10):2943-52.

70. van Driel PB, et al. Characterization and evaluation of the artemis camera for fluorescence-guided cancer surgery. Mol Imaging Biol. 2015;17(3):413-23.

71. Brouwer OR, et al. Feasibility of sentinel node biopsy in head and neck melanoma using a hybrid radioactive and fluorescent tracer. Ann Surg Oncol. 2012;19(6):1988-94.

72. Marshall MV, et al. Single-dose intravenous toxicity study of IRDye $800 \mathrm{CW}$ in Sprague-Dawley rats. Mol Imaging Biol. 2010;12(6):583-94.

73. de Valk KS, et al. First-in-human assessment of cRGD-ZW800-1, a zwitterionic, integrin-targeted, near-infrared fluorescent peptide in colon carcinoma. Clin Cancer Res. 2020;26(15):3990-8.

74. Crane LM, et al. Intraoperative near-infrared fluorescence imaging for sentinel lymph node detection in vulvar cancer: first clinical results. Gynecol Oncol. 2011;120(2):291-5.

75. Ishizawa $T$, et al. Real-time identification of liver cancers by using indocyanine green fluorescent imaging. Cancer. 2009;115(11):2491-504.

76. Purich K, et al. Intraoperative fluorescence imaging with indocyanine green in hepatic resection for malignancy: a systematic review and meta-analysis of diagnostic test accuracy studies. Surg Endosc. 2020;34(7):2891-903.

77. Hong G, Antaris AL, Dai H. Near-infrared fluorophores for biomedical imaging. Nat Biomed Eng. 2017;1(1):0010.

78. Joshi BP, Wang TD. Targeted optical imaging agents in cancer: focus on clinical applications. Contrast Media Mol Imaging. 2018;2018:2015237.

79. Hernot S, et al. Latest developments in molecular tracers for fluorescence image-guided cancer surgery. Lancet Oncol. 2019;20(7): e354-67.

80. Weissleder R, et al. In vivo imaging of tumors with proteaseactivated near-infrared fluorescent probes. Nat Biotechnol. 1999;17(4):375-8.

81. Choi HS, et al. Targeted zwitterionic near-infrared fluorophores for improved optical imaging. Nat Biotechnol. 2013;31(2):148-53.

82. Lamberts LE, et al. Tumor-specific uptake of fluorescent bevacizumab-IRDye $800 \mathrm{CW}$ microdosing in patients with primary breast cancer: a phase I feasibility study. Clin Cancer Res. 2017;23(11):2730-41.

83. Miller SE, et al. First-in-human intraoperative near-infrared fluorescence imaging of glioblastoma using cetuximabIRDye800. J Neurooncol. 2018;139(1):135-43.

84. van der Vorst JR, et al. Randomized comparison of near-infrared fluorescence imaging using indocyanine green and 99(m) technetium with or without patent blue for the sentinel lymph node procedure in breast cancer patients. Ann Surg Oncol. 2012;19(13):4104-11.

85. Hutteman M, et al. Randomized, double-blind comparison of indocyanine green with or without albumin premixing for nearinfrared fluorescence imaging of sentinel lymph nodes in breast cancer patients. Breast Cancer Res Treat. 2011;127(1):163-70.

86. Deguchi JO, et al. Inflammation in atherosclerosis: visualizing matrix metalloproteinase action in macrophages in vivo. Circulation. 2006;114(1):55-62.
87. Osborn EA, et al. Metabolic and molecular imaging of atherosclerosis and venous thromboembolism. J Nucl Med. 2017;58(6):871-7.

88. Kelderhouse LE, Mahalingam S, Low PS. Predicting response to therapy for autoimmune and inflammatory diseases using a folate receptor-targeted near-infrared fluorescent imaging agent. Mol Imaging Biol. 2016;18(2):201-8.

89. Wunder $\mathrm{A}$, et al. In vivo imaging of protease activity in arthritis: a novel approach for monitoring treatment response. Arthritis Rheum. 2004;50(8):2459-65.

90. Slooter MD, et al. Near infrared fluorescence imaging for early detection, monitoring and improved intervention of diseases involving the joint. Connect Tissue Res. 2015;56(2):153-60.

91. Verbeek FP, et al. Near-infrared fluorescence imaging of both colorectal cancer and ureters using a low-dose integrin targeted probe. Ann Surg Oncol. 2014;21(Suppl 4):S528-37.

92. Verbeek FP, et al. Intraoperative near infrared fluorescence guided identification of the ureters using low dose methylene blue: a first in human experience. J Urol. 2013;190(2):574-9.

93. Mahalingam SM, et al. Intraoperative ureter visualization using a novel near-infrared fluorescent dye. Mol Pharm. 2018;15(8):3442-7.

94. Diana M, et al. Prospective evaluation of precision multimodal gallbladder surgery navigation: virtual reality, near-infrared fluorescence, and X-ray-based intraoperative cholangiography. Ann Surg. 2017;266(5):890-7.

95. Nguyen QT, Tsien RY. Fluorescence-guided surgery with live molecular navigation-a new cutting edge. Nat Rev Cancer. 2013;13(9):653-62.

96. Whitney MA, et al. Fluorescent peptides highlight peripheral nerves during surgery in mice. Nat Biotechnol. 2011;29(4):352-6.

97. de Boer E, et al. Optical innovations in surgery. Br J Surg. 2015;102(2):e56-72.

98. Themelis G, et al. Real-time intraoperative fluorescence imaging system using light-absorption correction. J Biomed Opt. 2009;14(6):064012.

99. Nagengast WB, Hartmans E, Garcia-Allende PB, Peters FTM, Linssen MD, Koch M, Koller M, Tjalma JJJ, Karrenbeld A, Jorritsma-Smit A, Kleibeuker JH, van Dam GM, Ntziachristos V. Near-infrared fluorescence molecular endoscopy detects dysplastic oesophageal lesions using topical and systemic tracer of vascular endothelial growth factor A. Gut. 2019;68(1):7-10. https://doi.org/10.1136/gutjnl-2017-314953.

100. van der Poel HG, et al. Intraoperative laparoscopic fluorescence guidance to the sentinel lymph node in prostate cancer patients: clinical proof of concept of an integrated functional imaging approach using a multimodal tracer. Eur Urol. 2011;60(4):826-33.

101. Newton AD, Predina JD, Nie S, Low PS, Singhal S. Intraoperative fluorescence imaging in thoracic surgery. J Surg Oncol. 2018;118(2):344-55. https://doi.org/10.1002/jso.25149.

102. KleinJan GH, et al. Optimisation of fluorescence guidance during robot-assisted laparoscopic sentinel node biopsy for prostate cancer. Eur Urol. 2014;66(6):991-8.

103. Marano A, et al. Application of fluorescence in robotic general surgery: review of the literature and state of the art. World J Surg. 2013;37(12):2800-11.

104. Middelburg TA, et al. Correction for tissue optical properties enables quantitative skin fluorescence measurements using multi-diameter single fiber reflectance spectroscopy. J Dermatol Sci. 2015;79(1):64-73.

105. Brooks S, et al. Sources of variability in the quantification of tissue optical properties by multidiameter single-fiber reflectance and fluorescence spectroscopy. J Biomed Opt. 2015;20(5):57002. 
106. van Leeuwen-van Zaane F, et al. In vivo quantification of the scattering properties of tissue using multi-diameter single fiber reflectance spectroscopy. Biomed Opt Express. 2013;4(5):696-708.

107. Kruijt B, et al. In vivo quantification of chromophore concentration using fluorescence differential path length spectroscopy. J Biomed Opt. 2009;14(3):034022.

108. Liu R, et al. Current trends and key considerations in the clinical translation of targeted fluorescent probes for intraoperative navigation. Aggregate. 2021;2(3):e23.

109. Yoon $\mathrm{K}$, et al. A multi-detection fluorescence dye with 5-ALA and ICG using modified light emitting diodes. Curr Opt Photonics. 2019;3(3):256-62.

110. Zheng Y, Yang H, Wang H, Kang K, Zhang W, Ma G, Du $\mathrm{S}$. Fluorescence-guided surgery in cancer treatment: current status and future perspectives. Ann Transl Med. 2019;7(Suppl 1):S6. https://doi.org/10.21037/atm.2019.01.26.

111. Nagaya T, Nakamura YA, Choyke PL, Kobayashi H. Fluorescence-guided surgery. Front Oncol. 2017;7:314. https://doi.org/ 10.3389/fonc.2017.00314.

112. Pahang F, et al. Fluorescence properties of methylene blue molecules coupled with metal oxide nanoparticles. OSA Continuum. 2020;3(3):688-97.

113. Kallaway $\mathrm{C}$, et al. Advances in the clinical application of Raman spectroscopy for cancer diagnostics. Photodiagnosis Photodyn Ther. 2013;10(3):207-19.

114. Wang W, et al. Real-time in vivo cancer diagnosis using Raman spectroscopy. J Biophotonics. 2015;8(7):527-45.

115. Ji M, et al. Detection of human brain tumor infiltration with quantitative stimulated Raman scattering microscopy. Sci Transl Med. 2015;7(309):309ra163.

116. Jermyn $\mathrm{M}$, et al. Intraoperative brain cancer detection with Raman spectroscopy in humans. Sci Transl Med. 2015;7(274):274ra19.

117. Hollon TC, et al. Rapid intraoperative diagnosis of pediatric brain tumors using stimulated Raman histology. Cancer Res. 2018;78(1):278-89.

118. Moradi H, et al. Raman micro-spectroscopy applied to treatment resistant and sensitive human ovarian cancer cells. J Biophotonics. 2017;10(10):1327-34.

119. Minamikawa T, Harada Y, Takamatsu T. Ex vivo peripheral nerve detection of rats by spontaneous Raman spectroscopy. Sci Rep. 2015;5:17165.

120. Kumamoto $Y$, et al. Rapid and accurate peripheral nerve imaging by multipoint Raman spectroscopy. Sci Rep. 2017;7(1):845.

121. Masson LE, et al. Dual excitation wavelength system for combined fingerprint and high wavenumber Raman spectroscopy. Analyst. 2018;143(24):6049-60.

122. Laing S, Jamieson L, Faulds K, et al. Surface-enhanced Raman spectroscopy for in vivo biosensing. Nat Rev Chem. 2017;1:0060. https://doi.org/10.1038/s41570-017-0060.

123. Santos LF, et al. Fiber-optic probes for in vivo Raman spectroscopy in the high-wavenumber region. Anal Chem. 2005;77(20):6747-52.

124. Barroso EM, et al. Raman spectroscopy for assessment of bone resection margins in mandibulectomy for oral cavity squamous cell carcinoma. Eur J Cancer. 2018;92:77-87.

125. Aaboubout $Y$, et al. Specimen-driven intraoperative assessment of resection margins should be standard of care for oral cancer patients. Oral Dis. 2021;27(1):111-6.

126. Puppels GJ et al. Tissue sample analysis. International Publication Number WO 2017/126955 A1. 2017.

127. Puppels GJ et al. Optical probe for measuring a tissue sample. International Publication Number WO 2017/111576 A1. 2017.
128. Lizio MG, Boitor R, Notingher I. Selective-sampling Raman imaging techniques for ex vivo assessment of surgical margins in cancer surgery. Analyst. 2021;146(12):3799-809.

129. Schie I, Stiebing C, Popp J. Looking for a perfect match: multimodal combinations of Raman spectroscopy for biomedical applications. J Biomed Opt. 2021;26(8):080601. https://doi. org/10.1117/1.JBO.26.8.080601.

130. Lizio MG, et al. Combined total internal reflection AF spectralimaging and Raman spectroscopy for fast assessment of surgical margins during breast cancer surgery. Biomed Opt Express. 2021;12(2):940-54.

131. Bendau E, et al. Distinguishing metastatic triple-negative breast cancer from nonmetastatic breast cancer using second harmonic generation imaging and resonance Raman spectroscopy. J Biophotonics. 2020;13(7):e202000005.

132. Bocklitz TW, et al. Pseudo-HE images derived from CARS/ TPEF/SHG multimodal imaging in combination with Ramanspectroscopy as a pathological screening tool. BMC Cancer. 2016;16(1):534.

133. Chowdhury AU, et al. Second harmonic generation guided Raman spectroscopy for sensitive detection of polymorph transitions. Anal Chem. 2017;89(11):5958-65.

134. Kang JW, et al. Combined confocal Raman and quantitative phase microscopy system for biomedical diagnosis. Biomed Opt Express. 2011;2(9):2484-92.

135. Kong K, et al. Diagnosis of tumors during tissue-conserving surgery with integrated autofluorescence and Raman scattering microscopy. Proc Natl Acad Sci. 2013;110(38):15189-94.

136. Boitor R, et al. Clinical integration of fast Raman spectroscopy for Mohs micrographic surgery of basal cell carcinoma. Biomed Opt Express. 2021;12(4):2015-26.

137. Boitor R, et al. Automated multimodal spectral histopathology for quantitative diagnosis of residual tumour during basal cell carcinoma surgery. Biomed Opt Express. 2017;8(12):5749-66.

138. Schie IW, et al. Morpho-molecular signal correlation between optical coherence tomography and Raman spectroscopy for superior image interpretation and clinical diagnosis. Sci Rep. 2021;11(1):9951.

139. Mosca S, et al. Spatially offset Raman spectroscopy. Nat Rev Methods Primers. 2021;1(1):21.

140. Lauwerends LJ, et al. Real-time fluorescence imaging in intraoperative decision making for cancer surgery. Lancet Oncol. 2021;22(5):e186-95.

141. Harlaar NJ, et al. Molecular fluorescence-guided surgery of peritoneal carcinomatosis of colorectal origin: a single-centre feasibility study. Lancet Gastroenterol Hepatol. 2016;1(4):283-90.

142. Nishio N, et al. Optical molecular imaging can differentiate metastatic from benign lymph nodes in head and neck cancer. Nat Commun. 2019;10(1):5044.

143. Newton $\mathrm{AD}$, et al. Intraoperative fluorescence imaging in thoracic surgery. J Surg Oncol. 2018;118(2):344-55.

144. Boogerd LS, et al. Laparoscopic detection and resection of occult liver tumors of multiple cancer types using real-time near-infrared fluorescence guidance. Surg Endosc. 2017;31(2):952-61.

145. Puppels GJ, et al. Carotenoids located in human lymphocyte subpopulations and natural killer cells by Raman microspectroscopy. Cytometry. 1993;14(3):251-6.

146. Hubbard TJE, Dudgeon AP, Ferguson DJ, Shore AC, Stone N. Utilization of Raman spectroscopy to identify breast cancer from the water content in surgical samples containing blue dye. 2020. https://doi.org/10.1002/tbio.202000023.

147. Horsnell J, et al. Raman spectroscopy: a promising method of assessing axillary lymph nodes even in the presence of blue dye. Eur J Surg Oncol. 2010;36(11):1111. 
148. Grimbergen MCM, van Swol CFP, van Moorselaar RJA, Mahadevan-Jansen A, et al. Feasibility of Raman spectroscopy in vitro after 5-ALA-based fluorescence diagnosis in the bladder. $\mathrm{N}$ Stone Proc SPIE 6078, Photonic Therapeutics and Diagnostics II, 60781D. 2006. https://doi.org/10.1117/12.655616.

149. Grimbergen MCM, et al. Raman spectroscopy of bladder tissue in the presence of 5-aminolevulinic acid. J Photochem Photobiol, B. 2009;95(3):170-6.

150. Livermore LJ, Isabelle M, Bell IM, Edgar O, Voets NL, Stacey R, Ansorge O, Vallance C, Plaha P. Raman spectroscopy to differentiate between fresh tissue samples of glioma and normal brain: a comparison with 5-ALA-induced fluorescence-guided surgery. J Neurosurg. 2020;1-11. https://doi.org/10.3171/2020.5. JNS20376.

151. Horgan CC, Bergholt MS, Thin MZ, Nagelkerke A, Kennedy R, Kalber TL, Stuckey DJ, Stevens MM. Image-guided Raman spectroscopy probe-tracking for tumor margin delineation. $\mathbf{J}$ Biomed Opt. 2021;26(3):036002. https://doi.org/10.1117/1.JBO. 26.3.036002.

152. Horgan CC, et al. Integrated photodynamic Raman theranostic system for cancer diagnosis, treatment, and post-treatment molecular monitoring. Theranostics. 2021;11(4):2006-19.

153. Egloff-Juras C, et al. NIR fluorescence-guided tumor surgery: new strategies for the use of indocyanine green. Int J Nanomedicine. 2019;14:7823-38.

154. Carr JA, et al. Shortwave infrared fluorescence imaging with the clinically approved near-infrared dye indocyanine green. Proc Natl Acad Sci. 2018;115(17):4465-70.
155. Santos IP, et al. Implementation of a novel low-noise InGaAs detector enabling rapid near-infrared multichannel Raman spectroscopy of pigmented biological samples. J Raman Spectrosc. 2015;46(7):652-60.

156. Santos IP, et al. Raman spectroscopic characterization of melanoma and benign melanocytic lesions suspected of melanoma using high-wavenumber Raman spectroscopy. Anal Chem. 2016;88(15):7683-8.

157. Santos IP, et al. Improving clinical diagnosis of early-stage cutaneous melanoma based on Raman spectroscopy. Br J Cancer. 2018;119(11):1339-46.

158. Pal S, et al. DNA-enabled rational design of fluorescence-Raman bimodal nanoprobes for cancer imaging and therapy. Nat Commun. 2019;10(1):1926.

159. Andreou $C$, et al. Imaging of liver tumors using surfaceenhanced Raman scattering nanoparticles. ACS Nano. 2016;10(5):5015-26.

160. Wang C, et al. Advanced nanotechnology leading the way to multimodal imaging-guided precision surgical therapy. Adv Mater. 2019;31(49):1904329.

Publisher's note Springer Nature remains neutral with regard to jurisdictional claims in published maps and institutional affiliations. 